\title{
Evaluation du risque de génotoxicité de l'eau de la rivière Okpara à Kika au Bénin
}

\author{
C. KOUGBLENOU ${ }^{1}$, S. AZONBAKIN ${ }^{2 *}$, M. ACCROMBESSI ${ }^{3}$, B. AGUEMON $^{3}$, \\ M. ADJAGBA ${ }^{2}$, B. AWEDE ${ }^{4}$, M.P. AINA ${ }^{1}$, R.B. DARBOUX ${ }^{2}$ et A. LALEYE ${ }^{2}$ \\ ${ }^{1}$ Laboratoire des Sciences et Techniques de l'eau, Ecole Polytechnique,Université d'Abomey-Calavi, Bénin. \\ ${ }^{2}$ Laboratoire d'Histologie, Biologie de la Reproduction, Cytogénétique et Génétique Médicale, Faculté des \\ Sciences de la Santé, Université d'Abomey-Calavi, Bénin. \\ ${ }^{3}$ Unité de Santé Communautaire et d'Epidémiologie, Faculté des Sciences de la Santé, Université \\ d'Abomey-Calavi, Bénin. \\ ${ }^{4}$ Unité de physiologie humaine, faculté des sciences de la santé, Université d'Abomey-Calavi, Bénin. \\ "Auteur correspondant ; E-mail : simon.azonbakin@fss.uac.bj
}

\section{REMERCIEMENTS}

Nous remercions le centre béninois de recherche scientifique et de l'innovation (CBRSI) du ministère de l'enseignement supérieur et de la recherche scientifique pour avoir octroyé le financement nécessaire à la réalisation de cette étude.

\section{RESUME}

La pollution des eaux continentales constitue l'un des problèmes majeurs dans plusieurs régions du Bénin. La rivière Okpara et ses effluents sont soumis à de fortes pressions anthropiques liées aux intrants chimiques agricoles et aux micropolluants charriés. Cette eau de surface traverse Kika (commune de Tchaourou) où les riverains l'utilisent comme source d'eau de boisson. Afin d'évaluer les risques sanitaires encourus par cette population du fait de cette exposition, une étude cytogénétique à la recherche des dommages produits au matériel génétique ( $\mathrm{ADN}$ ) a été réalisée. Les micronoyaux $(\mathrm{MN})$ ont été recherchés et l'indice de prolifération cellulaire (IP) a été calculé chez les riverains habitant Kika, personnes exposées (PE) en comparaison avec des personnes non exposées (PNE). Le taux de micronoyaux est significativement plus élevé $(62,24 \pm 3,88)$ chez les PE (Kika) par rapport aux PNE $(2,92 \pm 0,39)$. Quant à l'indice de prolifération, elle est significativement plus faible $(1,50 \pm 0,04)$ chez les PE (Kika) que chez les PNE $(2,20 \pm 0,06)$. Ces résultats attestent du risque de génotoxicité lié à la consommation de l'eau de la rivière de Kika.

() 2017 International Formulae Group. All rights reserved.

Mots clés: Génotoxicité, micronoyaux, pollution, indice de prolifération cellulaire

\section{ABSTRAT}

The pollution of inland waters is one of the major problems in several regions of Benin. The Okpara River and its effluents are subjected to high anthropogenic pressures related to agricultural chemical inputs and transported micropollutants. This surface water crosses Kika (commune of Tchaourou) where residents use it as a source of drinking water. In order to assess the health risks incurred by this population as a result of this 
exposure, we conducted a cytogenetic study in search of damage to genetic material (DNA). We thus quantified the micronuclei (MN) and calculated the cell proliferation index (PI) in residents living in Kika, exposed persons (PE) in comparison with unexposed persons (PNE). Micronuclei levels were significantly higher $(62.24 \pm 3.88)$ in PE (Kika) compared to PNE $(2.92 \pm 0.39)$. The proliferation index was significantly lower $(1.50 \pm 0.04)$ in PE $(\mathrm{Kika})$ than in PNE $(2.20 \pm 0.06)$. These results attest to the risk of genotoxicity associated with the consumption of water from the Kika River.

(C) 2017 International Formulae Group. All rights reserved.

Keywords: Genotoxicity, Micronuclei, Pollution, Cell proliferation index.

\section{INTRODUCTION}

Les activités anthropiques sont responsables de l'introduction d'un grand nombre de substances chimiques dans l'environnement. L'eau, précieuse richesse de la nature indispensable à toute vie, fait l'objet d'une grande préoccupation de la plupart des pays en l'occurrence dans ceux en développement (OMS, 2004). Les cours d'eaux, notamment les lacs, constituent généralement le réceptacle de rejets d'origines industrielle, domestique et atmosphérique. Ils peuvent être également soumis à une pollution d'origine agricole par lessivage des sols. Ces rejets augmentent la charge en matières organiques des eaux superficielles et leur contamination peut se répercuter sur la chaîne alimentaire. Dans le cadre de la Directive Cadre Européenne sur l'eau, il est nécessaire d'évaluer l'impact de la contamination chimique sur les milieux aquatiques afin d'établir un diagnostic de l'état écologique des masses d'eau. Les biomarqueurs de génotoxicité permettent l'évaluation de l'impact des contaminants chimiques sur l'intégrité structurelle de l'ADN et sont utilisés comme indicateurs prédictifs d'effets au niveau populationnel (Alexandrescu, 2006; Sabharwal et al., 2015).

Au Bénin, les enjeux économiques liés à la production, et la commercialisation du coton ont largement motivé l'utilisation d'engrais chimiques et de pesticides dans ce secteur. Ces intrants agricoles chimiques ont des conséquences sanitaires importantes sur les populations utilisatrices. Actuellement, certaines molécules classées $1 \mathrm{~b}$ (hautement dangereux) ou $\mathrm{T}$ (toxiques) comme l'endosulfan ou le triazophos sont couramment utilisées avec des risques pour la faune, le sol, et les eaux de surface (Biaou, 2003; Toe, 2004; Adam 2010). La contamination des eaux de surface ou souterraines par les toxiques et les pesticides, en l'occurrence les fongicides, contenant des métaux lourds, peut s'ajouter aux contaminants déjà existants dans le sol (Cissé, 2003).

L'Okpara, un affluent du fleuve Ouémé, seule source d'approvisionnement en eau potable de la ville de Parakou, n'est pas épargnée de la pollution due à l'utilisation intensive de produits phytosanitaires pour l'amélioration des rendements agricoles. L'activité principale de la population riveraine à Kika (commune de Tchaourou) dans le bassin versant est la culture du coton et des produits vivriers. Elle nécessite l'utilisation intensive d'engrais chimiques et de pesticides herbicides ou fongicides (Adam, 2010 ; Ferron, 2006). Malgré la diversité de ses contaminants (pesticides et autres micropolluants), cette eau brute de l'Okpara demeure d'utilisation quotidienne par la population riveraine. L'objectif de ce travail est d'évaluer la génotoxicité liée à la consommation de l'eau de l'Okpara par la population riveraine exposée (PE) à Kika en 
comparaison avec une population non exposée (PNE) qui vit à Parakou et qui n'a aucun contact avec cette source d'eau.

\section{MATERIEL ET METHODES}

Il s'est agi d'une étude transversale, comparative et descriptive cas témoins. La population d'étude est constituée de deux groupes : les personnes consommant de façon permanente l'eau de la rivière Okpara qui constitue la population exposée (PE) et celles consommant uniquement l'eau potable de la Société Nationale des Eaux du Bénin (SONEB/Parakou) formant la population non exposée (PNE). Tous les individus ont été sélectionnés de façon aléatoire.

Les critères d'inclusion sont les suivants :

- pour les exposés : être habitant de Kika, être âgé de 25 à 50 ans et n'utiliser que l'eau brute de l'Okpara pour tout usage.

- pour les non exposés : être habitant de Parakou, être âgé de 25 à 50 ans, n'utiliser que l'eau de la SONEB/Parakou et n'avoir aucun contact avec l'eau de la rivière Okpara.

Pour les deux groupes, n'ont pas été incluses dans l'étude, les personnes :

- $\quad$ exerçant toute activité qui expose à des agents clastogènes : mécanicien garagistes, vendeurs d'hydrocarbures, coiffeurs, travailleurs des industries de cimenterie, de fabrique d'engrais, d'huilerie, de savonnerie ou des sites de concassage ... etc,

- malades ou sous traitement,

- fumeuses ou alcooliques,

- exposées aux radiations.

En tenant compte de la taille de la population de Kika , un arrondissement de la commune de Tchaourou, le calcul de la taille de l'échantillon avec la formule de Schwartz a permis de retenir 25 individus représentant un échantillon représentatif. Les deux groupes ont été appariés selon l'âge et le sexe et comptent chacun 25 personnes dont 13 hommes et 12 femmes.

Après un entretien individuel et après avoir donné son consentement éclairé, chaque personne subit un prélèvement de $5 \mathrm{ml}$ de sang veineux dans un tube hépariné. Ce prélèvement a servi à une recherche de micronoyaux (MN) suivant la technique de Fenech et Morley (Fenech, 1997). Ces micronoyaux sont des marqueurs de génotoxicité stables permettant d'évaluer de façon efficiente les effets génotoxiques des métaux lourds à long terme.

La lecture a été effectuée à l'objectif $X 100$ à immersion sur un microscope photonique Olympus BX 62 couplé à un analyseur d'image de type Cytovision (Applied Biosystems). Seuls les lymphocytes binucléés ont été pris en compte. Ne sont considérés comme micronoyaux que toutes entités nucléaires indépendantes des noyaux principaux, de même coloration, dont la taille est comprise entre le seizième et le tiers du plus petit des deux noyaux principaux. Pour chaque individu, 1000 lymphocytes binucléés sont examinés et les cellules portant au moins un micronoyau sont comptabilisées. Les résultats sont exprimés en nombre de micronoyaux pour 1000 lymphocytes binucléés observés.

L'indice de prolifération cellulaire (IP) a été calculé selon la formule de Titenko-Holland (Titenko-Holland, 1997) :

$$
\mathrm{IP}=\frac{(1 \times N 1)+(2 \times N 2)+(3 \times N 3)+(4 \times N 4)}{1000 \text { Cellules analysées }}
$$

$N 1$ : nombre de cellules mononucléées; $N 2$ : nombre de cellules binucléées ;

N3 : nombre de cellules trinucléées ; N4 : nombre de cellules tétranucléées.

L'analyse statistique a été effectuée grâce au logiciel Epi Info version 3.0.3 et dans l'environnement statistique $\mathrm{R} \quad(\mathrm{R}$ Core Development Team, 2014), version 3.0.3. Le 
seuil de significativité des tests a été fixé à $\alpha=5 \%$. Les coefficients de corrélation de Pearson ont été déterminés et leur significativité a été recherchée.

La présente étude, réalisée dans le cadre des travaux académiques, a été conduite dans le strict respect des règles de bonnes pratiques cliniques (GCP). Le consentement libre et éclairé des patients a été obtenu par écrit. La confidentialité a été rigoureusement respectée au cours de la collecte des données. Les informations obtenues dans le cadre de cette étude ont été traitées dans l'anonymat. Les résultats issus des différentes analyses ont été communiqués aux patients pour leur sensibilisation.

\section{RESULTATS}

$\mathrm{Au}$ total, 50 personnes dont 25 exposées à l'eau brute de l'Okpara et 25 autres non exposées ont été incluses dans chaque groupe. La population des exposés et des non exposés a été repartie de façon presque équitable entre les 2 sexes afin de bien apparier les membres de chaque groupe d'étude. Ainsi donc chaque groupe compte 13 hommes et 12 femmes.

L'âge moyen des personnes enquêtées est de 35,30 $\pm 1,01$ ans. Il est de 37,10 \pm 1,41 ans chez les PE et de $33,5 \pm 1,38$ ans chez les PNE. La différence entre les deux groupes n'est pas significative $(\mathrm{t}=1,82 ; \mathrm{DF}=48$; et $\mathrm{p}=0,075)$. L'homogénéité de l'âge entre la population non exposée et la population exposée est ainsi assurée.

Le test de micronoyaux (MN) et l'indice de Prolifération cellulaire (IP) ont été utilisés comme indicateurs biologiques des dommages produits à l'ADN suite à l'exposition aux agents génotoxiques.

La Figure 1 illustre une image microscopique d'un micronoyau que nous avions obtenu lors de cette étude. Les taux de MN obtenus dans les 2 populations sont résumés dans le Tableau 1.

Le taux de MN varie globalement de 0 à 7 chez les PNE et de 22 à 89 chez les PE. Le taux moyen de MN est considérablement plus élevé chez les PE $(62,24 \pm 3,88)$ que chez les PNE $(2,92 \pm 0,39)$. Cette différence est très significative $(p=0,001,<0,05)$. Ces résultats ont montré également une variabilité importante dans la réponse des PE.

Les résultats de l'indice de prolifération cellulaire (IP) calculé dans les deux groupes sont consignés dans le Tableau 2.

L'IP varie globalement de 1,81 à 2,93 chez les PNE et de 1,1 à 1,77 chez les PE. En effet, l'IP moyen dans la population exposée $(1,50 \pm 0,04)$ est moins élevé que dans la population non exposée $(2,20 \pm 0,06)$. Cette différence est hautement significative $(\mathrm{p}=0,003,<0,05)$.

Afin de mieux expliquer les différences observées entre les PE et les PNE, l'évolution des deux indicateurs biologiques en fonction de l'âge et du sexe a été étudiée. Les Figures 2 et 3 présentent le nombre de $\mathrm{MN}$ et l'IP en fonction de l'âge dans les deux populations. Chez les PE, plus l'âge avance, plus le taux de MN augmente alors que l'IP est plus faible. Les différences observées sont hautement significatives $(p=0.01,<0,05)$. Par contre, chez les PNE, l'âge n'affecte pas le taux des MN ou la cinétique de la division cellulaire.

Les Figures 4 et 5 présentent les variations des MN et de l'IP en fonction du sexe chez les PE et les PNE. Dans le groupe des PE, les taux moyens de MN sont plus élevés chez les femmes $(75,33 \pm 2,91)$ que chez les hommes $(50,31 \pm 0,09)$ contrairement à l'IP. Cette différence est significative $(\mathrm{p}=$ $0.002,<0,05)$. Par contre chez les PNE, le facteur sexe n'affecte ni l'IP ni les taux moyens de MN. La différence n'est pas significative. 


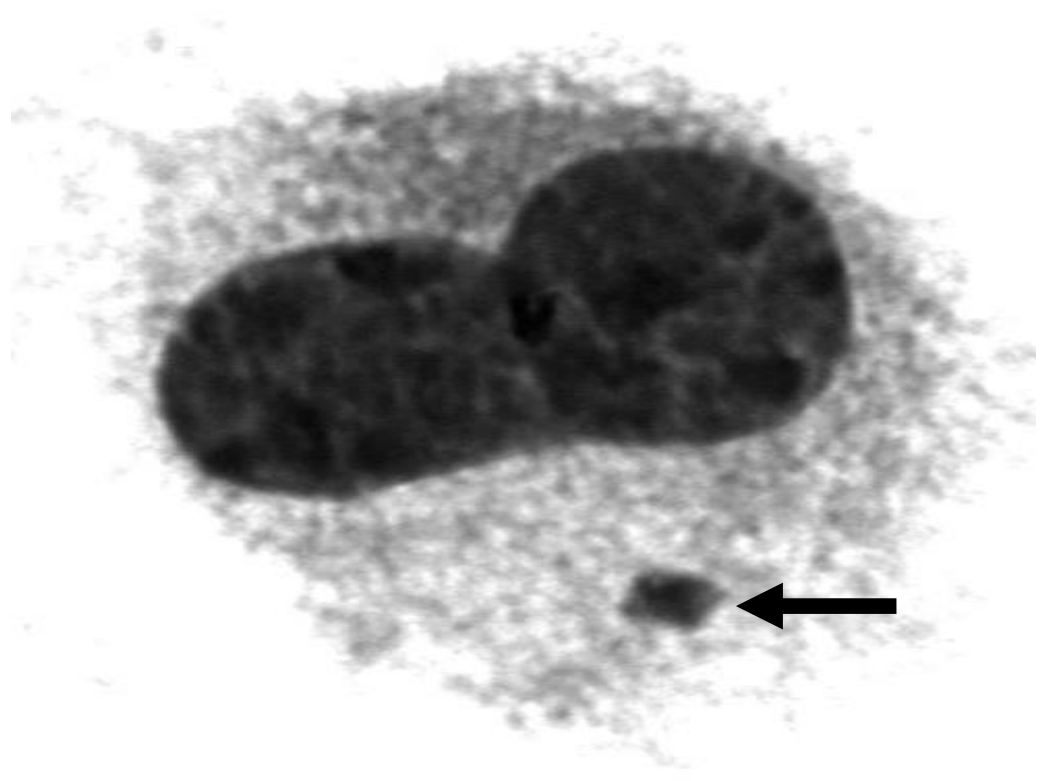

Figure 1 : Cellules en fin de mitose avec un micronoyau (Flèche) (x 400).

Tableau 1 : Moyenne des MN calculée chez les personnes exposées et les non exposées.

\begin{tabular}{lc}
\hline Groupes & Moyenne MN \\
\hline PNE & $2,92 \pm 0,39$ \\
\hline PE & $62,24 \pm 3,88$ \\
\hline PNE : Population non exposée & PE : Population exposée
\end{tabular}

Tableau 2 : Moyenne de l'indice de prolifération dans les deux populations d'étude.

\begin{tabular}{lc}
\hline Groupes & Moyenne de l'IP \\
\hline PNE & $2,20 \pm 0,06$ \\
\hline PE & $1,50 \pm 0,04$ \\
\hline PNE : Population non exposée ; PE : Population exposée $\quad ;$ IP : Indice de Prolifération
\end{tabular}




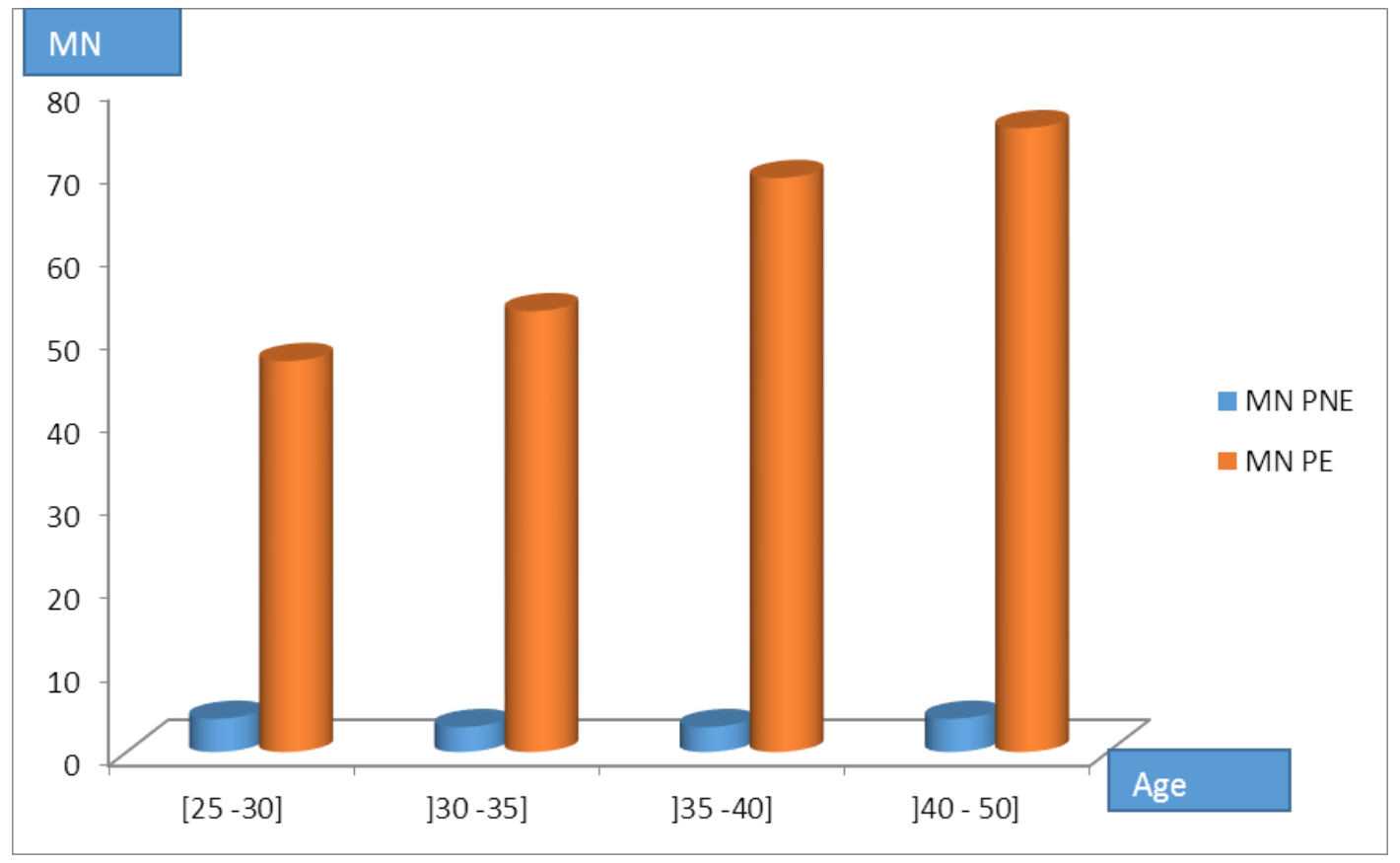

Figure 2 : Influence de l'âge sur le taux de $\mathrm{MN}$ dans les deux populations. PNE : Population non exposée PE : Population exposée MN : Micro noyaux.

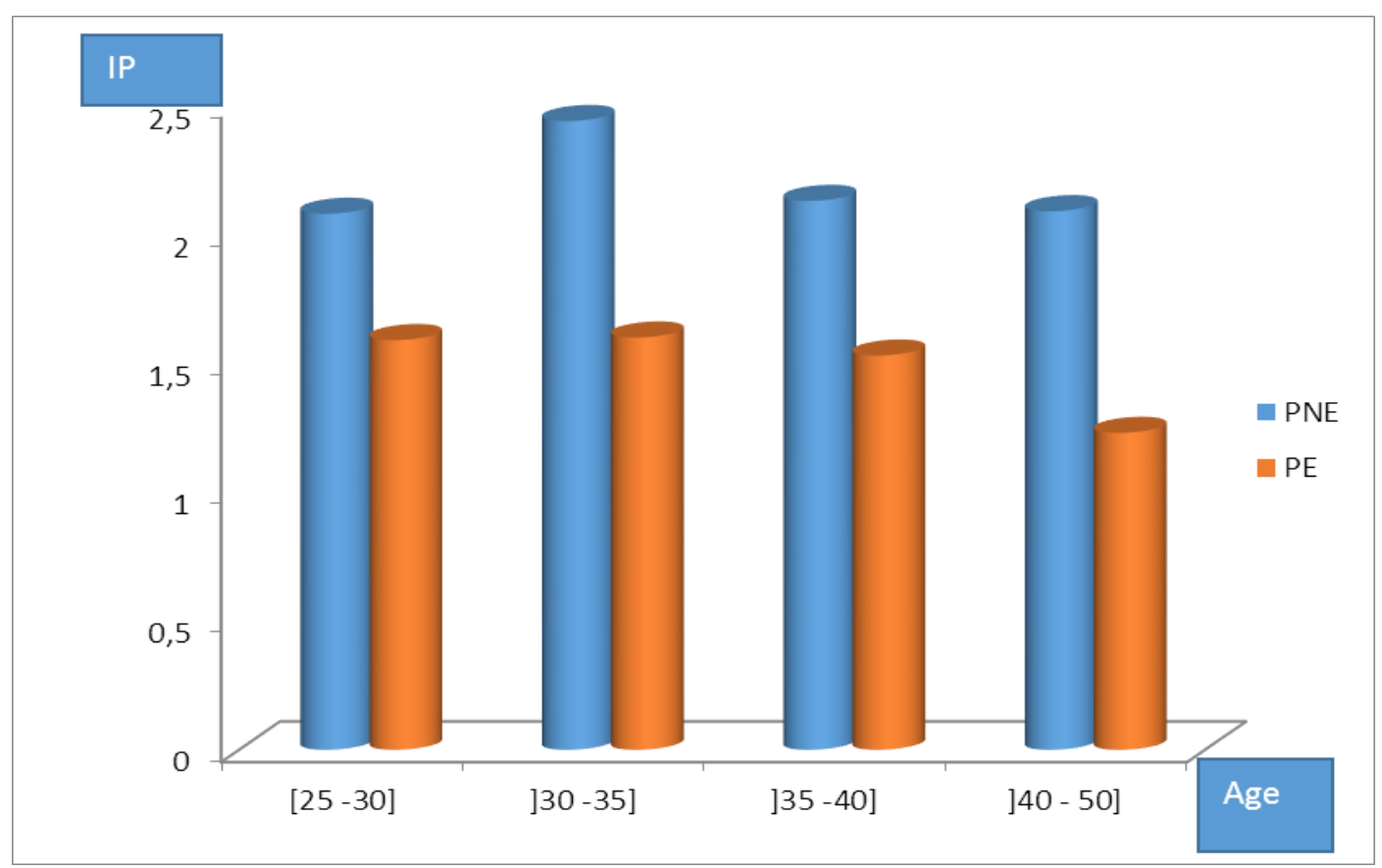

Figure 3 : Influence de l'âge sur l'IP dans les deux populations d'étude. IP : Index de Prolifération , PE : Population exposée , PNE : Population non exposée. 


\section{MN}

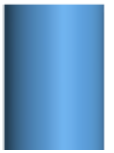

PE

\section{PNE}

- Feminin

- Masculin

Figure 4 : Influence du sexe sur le taux des MN chez les PE et les PNE. MN : Micro noyaux , PE : Population exposée , PNE : Population non exposée.

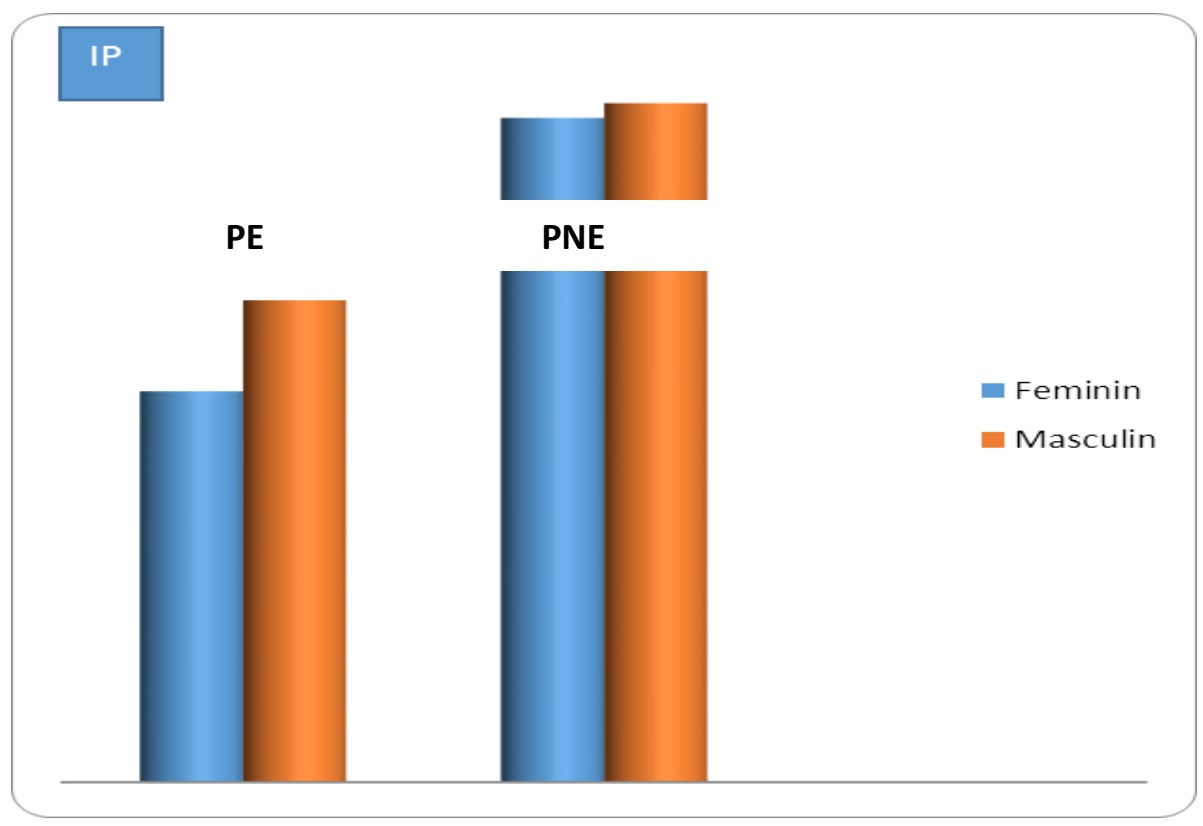

Figure 5 : Influence du sexe sur l'IP dans les deux populations.

PE : Population Exposée ; PNE : Population non Exposée ; IP : Index de Prolifération. 


\section{DISCUSSION}

L'introduction dans les cours d'eau (lacs, fleuves et rivières) de déchets ménagers ou industriels, affecte les écosystèmes aquatiques et impacte négativement la santé humaine. Parmi ces déchets, les métaux toxiques issus de la contamination liée à une pollution du fait des activités humaines occupent une bonne place. Ces déchets ou leurs métabolites constituent souvent des agents mutagènes agissant à plus ou moins longue échéance. $\mathrm{La}$ recherche de micronoyaux (MN), constitue un marqueur biologique précoce de la génotoxicité de ces agents mutagènes. Les micronoyaux ont pour origine des fragments acentriques de chromosomes (c'est à dire dépourvus de centromère) ou des chromosomes entiers incapables de migrer vers les pôles de la cellule au cours de l'anaphase. Le test des micronoyaux permet de détecter l'activité de substances chimiques clastogènes et aneugènes chez l'homme après exposition (Bonassi, 2001; Fenech, 2003; Norppa, 2003, Glouib et al., 2006) . Dans la population de cette étude, il y a 30 fois plus de micronoyaux chez les exposés que chez les non exposés $(62,24 \pm 3,88$ et $2,92 \pm 0,39$ respectivement chez PE et PNE). Il existe donc une relation entre la consommation de l'eau de l'Okpara et l'occurrence des MN. Le nombre moyen de MN observé chez les PE est largement supérieur à celui trouvé par Glouib et al. (2006) au Maroc qui avaient étudié l'induction des micronoyaux et l'indice de la prolifération cellulaire dans la population des Mzamza exposée aux eaux usées. Dans leurs travaux, le taux moyen des MN était de 16 chez les PE et 3 chez les PNE.

La population de Kika riveraine de l'Okpara est fortement exposée aux effets génotoxiques étant donné qu'elles utilisent l'eau brute sans aucun traitement préalable. En effet, pour $20 \%$ des PE utilisant l'eau depuis 10 ans, la moyenne de MN est de 20 à 40. Pour les habitants exposés depuis longtemps, plus de 15 à 20 ans, les MN varient de 70 à 90 . Ainsi, l'induction des MN serait-elle liée à la durée d'exposition. Ces résultats corroborent les travaux de Michel (Michel, 2011) qui a montré que l'expression de cassures de l'ADN et la fréquence des micronoyaux dépendent de la saison et de la durée d'exposition aux eaux contaminées par les substances aneugènes.

$\mathrm{La}$ fréquence de MN augmente significativement avec l'âge $(\mathrm{p}=0,033)$ et est également liées au sexe $(\mathrm{p}=0,002)$. L'occurrence des $\mathrm{MN}$ est particulièrement élevée chez les femmes. Ces résultats se rapprochent de ceux de Glouib (Glouib et al., 2006). Les femmes étant plus en contact avec l'eau pour leurs activités ménagères sont plus exposées aux effets nocifs des polluants charriés.

Les lésions de l'ADN générées par les agents génotoxiques et les mutations qu'elles peuvent induire dans les cellules somatiques et germinales sont susceptibles d'entraîner l'apparition de pathologies chez les personnes exposées et leur descendance. Bien que ce ne soit pas la seule pathologie pouvant résulter d'une atteinte de l'ADN, le cancer reste la principale hantise quant aux expositions aux agents génotoxiques. Actuellement, d'autres marqueurs biologiques tels que les aberrations chromosomiques structurales (ACS) et les échanges de chromatides sœurs (SCE) sont évalués pour étudier la génotoxicité. Par ailleurs, l'accumulation de mutations contribue également au processus de vieillissement des populations, à la survenue des maladies neurodégénératives et cardiovasculaires et à l'induction de malformations et de pathologies diverses dans la descendance des personnes exposées (Bonassi, 2001 ; Neri, 2003 ; Holland, 2008). Dans cette étude le marqueur de cytotoxicité utilisé est le test des micronoyaux parque c'est un test stable, reproductible et spécifique des effets clastrogènes causés par les métaux lourds de l'environnement (Fenech, 2003).

L'évaluation de la cytotoxicité se fonde sur l'indice de prolifération des cellules dont 
la division cytoplasmique a été bloquée (IP). Il existe donc une corrélation inverse très significative entre l'IP et le taux des MN. (Corrélation $=-0,901$ et $p=0,001<0,05)$. En effet, lorsque le taux des MN augmente, la vitesse de la division cellulaire est retardée, ceci est probablement dû à l'activation des points de contrôle du cycle cellulaire en réponse aux lésions induites au niveau de l'ADN (Botta et al., 2006).

L'organisme possède des mécanismes de protection cellulaire qui protègent l'ADN et empêchent l'apparition de mutations. Le franchissement de cette première barrière de protection déclenche l'induction de signaux, tels ceux de la voie de signalisation MAP kinase. Ces signaux conduisent à l'arrêt du cycle cellulaire aux points de contrôle et à l'élimination des lésions de l'ADN. Ces mécanismes de protection efficaces permettent le maintien de taux de mutations proches des taux spontanés lors de l'exposition à de faibles doses (Norppa, 2003 ; Sabharwal et al., 2015). Ce n'est que lorsque ces mécanismes de protection cellulaire sont saturés dans le cas d'expositions à de plus fortes doses que le taux de mutation est augmenté.

Au total, au moyen du test des MN et celui de l'IP, la génotoxicité liée à la consommation de l'eau de l'Okpara par la population de Kika au Nord du Bénin a été mise en évidence. Ces marqueurs biologiques constituent un moyen important d'évaluation des risques de génotoxicité chez l'homme et sont déjà utilisés en routine dans plusieurs pays développés (Palanikumar, 2011).

\section{Conclusion}

La génotoxicité de la consommation d'eau brute de la rivière Okpara est patente ; mais l'importance et la gravité des conséquences pour la santé restent actuellement difficiles à prévoir. Une surveillance sanitaire structurée et à plus ou moins long terme de la population exposée s'impose, à charge pour les autorités de fournir une alternative sécurisée en eau potable. De plus, une étude plus large permettra de mieux apprécier l'impact et la nature des agents génotoxiques présents dans l'eau de l'Okpara. Tout porte à espérer que ce travail puisse inciter les décideurs à tous les niveaux à prendre les mesures adéquates pour l'accès à l'eau potable aux populations même celles des hameaux les plus reculés du Bénin.

\section{CONFLIT D'INTERETS}

Il n'existe aucun conflit d'intérêt entre les auteurs de ce manuscrit.

\section{CONTRIBUTIONS DES AUTEURS}

Les auteurs SA et CK ont contribué de façon équitable à ce travail. MA, BA ont assuré l'analyse statistique du travail.

MAD, s'est occupé de la coordination du plateau technique d'analyse des échantillons. BAW, AL, MPA, RBD ont assuré la coordination scientifique de ce travail de recherche académique. Tous les auteurs ont participé à la rédaction de l'article et ont donné leur correction.

\section{REMERCIEMENTS}

Nous remercions également habitants de Kika et de Parakou qui ont participé à cette étude.

\section{REFERENCES}

Adam S, Edorh PA, Totin H, Koumolou L, Amoussou E, Aklikokou K, and Boko M. 2010. Pesticides et métaux lourds dans l'eau de boisson, les sols et les sédiments de la ceinture cotonnière de Gogounou, Kandi et Banikoara (Bénin). Int. J. Biol. Chem. Sci., 4(4): 1170-1179.

Alexandrescu I, Havarneanu D, Popa D. 2006. New approaches in biomonitring human populations exposed to genotoxic agents: Epithelial cell micronucleus assay. $J$ Preventive Med., 14: 57-65. 
Biaou C, Alonso S, Truchot D, Abiola FA, Petit C. 2003. Contamination des cultures vivrières adjacentes et du sol lors d'une pulvérisation d'insecticides sur des champs de coton: cas du triazophos et de l'endosulfan dans le Borgou (Bénin). Revue Méd. Vét., 154(5): 339-344.

Bonassi, S., Fenech, M., Lando, C. 2001. Human Micronucleus project: international database comparison for the results with the cytokinesis-block micronucleus assay in human lymphocytes: effect of laboratory protocol, scoring criteria, and host factors on the frequency of micronuclei. Environ. Mol. Mutagen., 37 : 31-45.

Botta A, et Mathis J.2006. De la génotoxicité à la cancérogenèse. 29è Congrès national de médecine et santé au travail. Arch Mal Prof Env. , 67(2): 295-314.

Cisse IA, Tandia ST, Fall EH, Diop S. 2003. Usage incontrôlé des pesticides en agriculture périurbaine: cas de la zone des Niayes au Sénégal. Cahiers Agricultures., 12: 181-186.

Fenech M. 1997. The advantages and disadvantages of the cytokinesis-block micronucleus method. Mutat Res., 392(1-2): 11-8.

Fenech M, Chang WP, Kirsch-Volders M, Holland N, Bonassi S, Zeiger E. 2003. HUMN project: detailed description of the scoring criteria for the cytokinesisblock micronucleus assay using isolated human lymphocyte cultures. Mutat. Res., 534, 65-75.

Ferron P, Deguine JP, Mouté JE. 2006. Évolution de la protection phytosanitaire du cotonnier: un cas d'école. Cah. Agric., 15(1): 128-134.

Glouib K, Charaf B, El Kettani S, Hilali A. 2006. Induction des micronoyaux et indice de la prolifération cellulaire chez la population des mzamza exposée aux eaux usées. Lebanese Science Journal, 7(2): 3-12.

Holland N, Bolognesi C, Kirsch-Volders M, Bonassi S, Zeiger E, Knasmueller S. The micronucleus assay in human buccal cells as a tool for biomonitoring DNA damage: The HUMN project perspective on current status and knowledge gaps. Mutat Res., 659: 93-108.

Michel C. 2011. Biomarqueurs de génotoxicité chez Dreissena polymorpha: indicateurs de la pression chimique urbaine et variabilité naturelle des lésions de l'ADN. Thèse de Doctorat en Géosciences et Ressources Naturelles, option Ecotoxicologie. Soutenu le 19 Décembre 2011. Université Pierre et Marie Curie - Paris VI, 218 p

Migliore L, Coppedè F, Fenech M and Thomas P. 2011. Association of micronucleus frequency with neurodegenerative diseases. Mutagenesis, 26(1): 85-92. doi: 10.1093/mutage/geq067.

Neri M, Fucic A, Knudsen L, Lando C, Merlo, F, Bonassi S. 2003. Micronuclei frequency in children exposed to environmental mutagens: a review. Mutat. Res., 544: 243-254.

Norppa H, Falck GC. 2003. What do human micronucléi contain? Mutagenesis, 18(3) : 221-33.

Organisation Mondiale de la Santé. 2004. Directives pour l'Eau de Boisson (3ème Edn, vol.1). OMS : Genève ; 111p.

Palanikumar L, Panneerselvam N. 2011. Micronuclei assay: A potential biomonitoring protocol in occupational exposure studies. Genetika, 47(9) : 11691174. 
Pazou EYA, Laldyd P, Boko M, Van Gestel

CAM, Ahissou H, Akpona S, Van Hattum B, Swart K, Van Straalen NM. 2006. Contamination of fish by organochlorine pesticide residues in the Ouémé River catchment in the Republic of Bénin. Environment International, CODEN ENVIDV, 32(5): 594-599.

Sabharwal R, Verma P, Syed MA, Sharma T, Subudhi SK, Mohanty S, Gupta S. 2015. Emergence of micronuclei as a genomic biomarker. Indian J Med Paediatr Oncol., 36(4): 212-218. doi: 10.4103/0971-5851.171541

Titenko-Holland N, Windhan G, Kolachana P, Reinish F, Paravatham S, Osorio AM, Smith MT. 1997. Genotoxicity of malathion in human lymphocytes assessed using the micronucleus assay in vivo and in vitro: a study of malathionexposed workers. Mut. Res., 388: 85-95.

Toe AM, Kinane ML, Kone S, Sanfo-Boyarm E. 2004. Le non-respect des bonnes pratiques agricoles dans l'utilisation de l'endosulfan comme insecticide en culture cotonnière au Burkina Faso: quelques conséquences pour la santé humaine et l'environnement. Revue Africaine de Santé et de Production Animales, 2: 275-278. 